\title{
Towards an ontological modelling of preference relations
}

\author{
Daniele Porello $\bowtie$ and Giancarlo Guizzardi \\ Free University of Bozen-Bolzano, Italy \\ \{firstname. lastname $\}$ @unibz.it
}

\begin{abstract}
Preference relations are intensively studied in Economics, but they are also approached in AI, Knowledge Representation, and Conceptual Modelling, as they provide a key concept in a variety of domains of application. In this paper, we propose an ontological foundation of preference relations to formalise their essential aspects across domains. Firstly, we shall discuss what is the ontological status of the relata of a preference relation. Secondly, we investigate the place of preference relations within a rich taxonomy of relations (e.g. we ask whether they are internal or external, essential or contingent, descriptive or nondescriptive relations). Finally, we provide an ontological modelling of preference relation as a module of a foundational (or upper) ontology (viz. OntoUML).

The aim of this paper is to provide a sharable foundational theory of preference relation that foster interoperability across the heterogeneous domains of application of preference relations.
\end{abstract}

Keywords: Preference Relations, Ontology of Relations, Foundational Ontology, Conceptual Model, OntoUML

\section{Introduction}

Preference relations have been extensively studied in Economics as they provide an essential theory to formalise and possibly explain human economic behaviour. Preference relations are also ubiquitous in Computer Science, in particular in AI, Knowledge Representation, and Multiagent Systems, as they provide a key concept in domains such as planning, autonomous agents modellisation, recommender systems, and resource allocation. Moreover, the use of preference relations is very extensive in Conceptual Modelling, for instance in applications to domains such as business process, services, and economic exchange.

Although preference relations are an ubiquitous concept in many areas, a clear ontological foundation of them is still to be developed. In this paper, we start the program of providing an exhaustive ontological analysis of preference relations. In particular, we intend to provide an analysis of preference relations that integrates a theory of preference relations with a larger theory of the main ontological categories, by initiating the development of a module for preference 
relations to be integrated within a foundational (or upper) ontology (cf. [2, 9]). A foundational ontology is a theory of the most general categories that are required in heterogeneous modelling tasks and whose principles hold across-domain. The foundational ontology program is a quite interdisciplinary endeavour that requires cognitive adequacy, philosophical awareness, as well as effective formalisations. Examples of such very general categories are object, event, property, relation, state, process, space, and time.

The mathematical treatment of preference relation is very well understood as well as their empirical foundation. Preference relations usually involve an agent who provides a ranking (a type of ordering) among the available options or alternatives.

An essential distinction between preference types is that between ordinal vs cardinal preference. Ordinal preference are simply a (type of) ranking of the options, whereas cardinal preferences associate to each option a value that represents the level of satisfaction of the agent concerning that option. A formalisation of ordinal preference relation in terms of modern formal logic has been developed by the Nobel Prize for Economics Kenneth Arrow [1], who was inspired by the axiomatic method of Tarski. Preference relations are modelled by means of binary relations that satisfy a number of axioms, usually related to the theory of orders (e.g. total, partial, or weak orders). That is, preference relations can be axiomatised within a first-order theory of orders.

The foundation of cardinal preference relation is traditionally referred to the work of von Neumann and Morgenstern about the theory of expected utility [29]. In general, cardinal preference relations are usually modelled by means of utility functions defined on a set of alternatives with values in a suitable set of numbers (usually, the real numbers).

Traditionally, preference relations are ultimately founded on the choice behaviour of an agent, see [10]. Cardinal preferences can be rationalised, under suitable conditions, by ordinal preference, and ordinal preferences can in turn be derived from choice functions that meet a number of suitable conditions. This type of foundation grounds a preference relation, which is allegedly something that is in the mind of an agent, in an observable behaviour, that is, the agents' actual observable choices. This operationalisation of the concept of preference relations was one of the most salient contributions of the economic foundation of preference, justifying the empirical elicitation of human agents' preferences. Another important distinction between preferences depends on whether they are ascribed to individual agents or to collective agents. Collective preferences are important when modelling preferences ascribed to societies, groups of agents, or sets of users (e.g. in recommender systems). The foundation in Economics of collective preference is essentially due to [1]. A discussion of the formalisation of collective preferences in terms of a first-order theory of ordering is proposed in [17].

A recent approach to the foundation of preference relations has been developed by Dietrich and List [5,4] and discussed in [15,19]. Here, preference relations are dependent on the properties of the alternatives at issue, whose 
salience depends on the views of the agent. This view brings us close to the approach of this paper in that the properties of the preferences are intended in an ontological sense. Thus the work of Dietrich and List is one of the closest related work to the present contribution.

Although the formalisation of preference relations is quite well established, a detailed ontological understanding is missing. We intend to fill this gap by initiating the investigation of the ontological foundation of preference relations.

The methodology of this paper is the following. We start by analysing the semantics of sentences of the form "agent $i$ prefers $a$ over $b$ ". That is, we look for those pieces of reality that are required in order to make the statements about preferences true. Specifically, we investigate what are the truth-makers, see for instance [12], of the statements about preferences. This view has been introduced for the ontological analysis of relations in [7]. This approach naturally leads to asking important ontological questions about which type of objects are related by a preference relation, to what extent preference relations depends on the ontological properties of the relata, which type of relations is the type of preference relation.

The contribution of this paper is twofold. Firstly, we informally analyse the type of preference relations by discussing their place within a taxonomy of relations. Secondly, we propose a formalisation of our analysis by means of an ontologically well-founded approach to conceptual modelling. In particular, we extend the methodology of OntoUML, which provides a solid ontologically deep approach to conceptual modelling [9], to provide a formal ontological understanding of preference relations. Our aim is to develop a module of OntoUML for preference relations that explicitly manifests the truth-makers of preferential statements and relates preference relations to the main general categories of a foundational ontology. Finally, we discuss how to model various types of preference relations by extending our proposed OntoUML module.

The remainder of this paper is organised as follows. Section 2 discusses an informative taxonomy of relations. Section 3 proposes how to place preference relations within the taxonomy of relations and discusses what may counts as a truth-maker of a relational sentence involving preferences. Section 4 proposes the modelling of preference relations in OntoUML and presents viable extensions. Section 5 concludes.

\section{A taxonomy of relations}

We summarise the discussion of the kinds of relations in terms of their truthmakers. To provide a fine-grained analysis of relations, we introduce three pairs of distinction that were traditionally at the core of important debates in analytical philosophy on the nature of relations: internal and external, essential and contingent, and descriptive and non-descriptive relations. We summarise the examples of this types of relations in Table 1.

Before proposing the definitions of those distinctions, we have to introduce a number of important concepts in ontology to categories properties. The choice 
of terminology we use in this paper is mainly due to [7]. A formalisation of the subsequent concepts in first-order logic is presented in [2, 9, 8, 22].

An individual quality, or simply a quality, is a property of an object, usually used to make comparisons between objects along a certain dimension. We say that the quality inhere in its object, which is termed the bearer of the quality. The relations of inherence is a particular type of existential dependence, which has been formalised in [9]. Examples of qualities of an object are the color of this rose or the weight of this laptop. We say that the quality existentially depends on its bearer, in the sense that the color of a certain rose is always ontologically separated from the color of a different rose. To compare qualities of different objects, we make the distinction between the qualities of an objects and the values of those qualities, i.e. the quality values (or qualia). The quality values are abstract objects that are introduced for the purpose of explaining or measuring the similarity of two objects along a certain dimension (e.g. the color). Quality values are possibly organised into metric spaces with a distance function that captures the similarity judgments between quality values. Examples of such metric structures are Gärdernfors conceptual spaces, cf [6].

Relational qualities (or externally dependent modes in [9]) are qualities whose existence depends (besides on the bearer of the quality) on an object which is ontologically separated to the object in which they inhere. ${ }^{1}$ Examples of relational quality are Mary's desire of buying a Ferrari which existentially depends on Mary, but also on the object of the desire. In Section 4, we shall discuss quality values also for relational qualities.

We term a quality intrinsic of an object if and only if it does not depend on any other object besides its bearer. For instance, one may view the color of an object as a quality that only depends on the object.

An essential property of an object is an intrinsic property that depends on the mere existence of the object. For example, if one views that any existing object must have a mass, then the mass is an essential property of any object.

We move now to the definitions of types of relations. We start by recalling the distinction between internal and external relations. We use a first-order notation for relations, where $R\left(a_{1}, \ldots a_{n}\right)$ denotes an $n$-ary relation. We say that $R$ is internal if and only if its truth-value only depends on the intrinsic properties of its relata; a relation is external if that is not the case. ${ }^{2}$ For instance, the truthvalue of " $a$ is taller than $b$ " depends on the height of $a$ and $b$, which are intrinsic properties of $a$ and $b$, whereas the truth-value of " $a$ desires $b$ " does not depend only on intrinsic properties of $a$ and $b$, hence it is external.

\footnotetext{
${ }^{1}$ We do not have space here to provide an exhaustive view of the separation of objects. We simply say that two objects are separated if they are existentially independent, cf $[9]$.

2 This is the definition provided by Russell [25]. A stronger definition is the one proposed by Moore [14], which views an internal relation as a relation the holds merely in virtue of the existence of the relata. This second view of internal relation is what we term here essential, following the terminology of [7].
} 
A relation is essential if and only if its truth-value depends only on the existence of its relata; it is contingent otherwise. For instance, " $a$ is taller than $b$ " is not essential, since the height is not an essential property of the object, e.g. there may be moments in time where a person $b$ is taller than a person $a$. By contrast, if one views that any object, in virtue of its mere existence, must have a mass, then " $a$ is heavier than $b$ " is an essential relation.

Finally, we introduce the distinction between descriptive and non-descriptive relations, which have been investigated in particular in [7].

A relation is descriptive if and only if its truth-value depends on at least one property of some of its relata which is existentially dependent on another distinct relata; it is non-descriptive otherwise. For instance, in a comparative relation between objects, as in the case of " $a$ is taller than $b$ ", the height differential that makes $a$ taller depends on $b$ (that is, it is externally dependent on $b$ ), thus the relation is descriptive. By contrast, a comparative relation between mere quality values is non-descriptive: according to this view, that the number associated to the height of $a$ is bigger or smaller than the number associated to the height of $b$ only depends on the numbers themselves.

The following table summarises the examples of the types of relations that we have introduced. As the table shows, descriptive vs non-descriptive and internal vs external provides the two dimensions for analysing relations. Moreover, one may find essential relations in any possible combination of the previous criteria. For the relationship between this taxonomy of relations and the problem of reifications of relations, that is, the problem of discussing which type of relations deserves reification, we refer to [7].

In the next section, we shall discuss which is the suitable categorsation for preference relations.

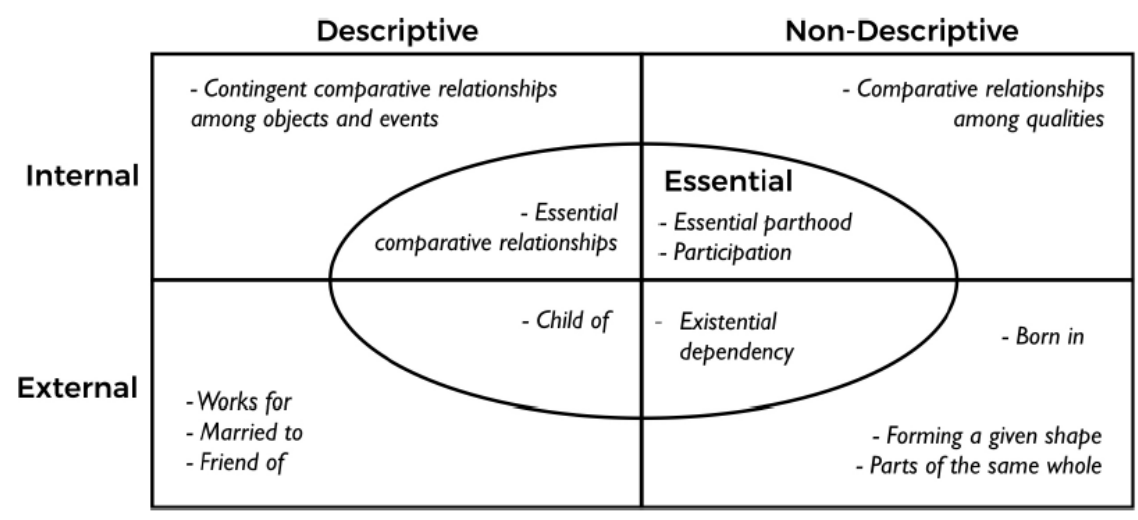

Table 1. Types of relations 


\section{$3 \quad$ The type of preference relations}

We discuss now what is the placement of preference relations within the taxonomy of relations discussed in the previous section. At the end of this section, we apply the categorisation of preference relations in order to elicit the truthmakers of statements about preferences. We focus on sentences such as ' $i$ prefers $a$ to $b "$, where $i$ is an agent and $a$ and $b$ are alternative options. In this section, we abstract from the type of preference relation, e.g. whether they are cardinal or ordinal preferences, or individual or collective preferences, and we aim to approach any preference relation.

Preference relations are in fact ternary relations, we denote them by means of the formula $\operatorname{PREF}(i, a, b)$. The first component of the preference relation $i$ is an agent, thus the property of agency must be spelled our in order to provide an exhaustive ontological model of a preference relation. Here, we simply assume that the user, or the modeller, can endorse any vision of agency she or he may see fit (e.g. the belief-desire-intention model of agency [23,3]). In the case of collective preferences, that is where $i$ is a collective agents or a community of agents, we need to choose a view of collective agency to complement the ontological model of preference, e.g. [11,21,20].

The type of the other two components of the preference relation is in fact quite general. Preference relations may be defined on very heterogeneous types of entities. For instance, an agent may have preferences over objects, candidates for elections, parties, vacation plans, evening events, services, loans or mortgages, stock options, and so on. Preference relation are indeed an ubiquitous concept in a variety of economic settings. Thus, in principle, we do not want to put any substantial restriction on the domain of the possible pairs of entities that may enter the second and the third component of the preference relation.

Concerning the ontological status of the truth-makers of a statement about preference, we notice that the qualities of the entities $a$ and $b$ are indeed not sufficient for assessing whether $a$ is preferred to $b$ or not. For instance, a blue car may be preferred to a red one by a certain agent, whereas the opposite may hold for another agent. Moreover, the preferences of an agent may evolve through time, may depend on what an agent already owns, may depend on the resources available to the agent. This examples shows that we have to access the view that the agent $i$ has about the options $a$ and $b$, in order to assess the truth-value of the preference relation.

To understand the relationship between the options involved in a preferential statement and the agent, we introduce the concepts of value, value magnitude, value entity (or value bearer), and value beholder, cf [28].

A value beholder is a role that an agent plays qua agent who is making a choice, or assessing a preference, between two options ${ }^{3}$. The value entity is again a role, this time, it is the role that an option plays in the context of a preference assessment of an agent. The value entity is a role that depends on an agent $i$ and on the option at issue. For simplicity here, we assume that the value

\footnotetext{
${ }^{3}$ For an ontological theory of roles, we refer to [9] and [13]
} 
entity of an option for an agent is unique, thus we refer to it by the definite description "the value entity of $a$ for $i " .{ }^{4}$ The category of value is intended here as a category of qualities that inhere in the value beholder. An element of this category represents the value of the value entity for the value beholder.

The value magnitude is an abstract category whose function is to enable comparisons between the values that agents associate to value entities. ${ }^{5}$ We may assume here that this category as an abstract set of ordered points. In treating preference relation, we are assuming that relational qualities (such as the value of a value entity for a value beholder) have also quality values. In particular, the quality value of the value of an option for an agent $i$ is an element of the category of value magnitude. To formalise this view, we may introduce a relation of location between the relational qualities (the category of value) involved in a preference statement and the space of values (the category of value magnitude). ${ }^{6}$

For instance, in the context of the statement "Mary prefers a Porsche to a Ferrari", Mary plays the role of the value beholder, the options Porsche and Ferrari are playing the role of the value entities of this context, the values of this value entities for Mary (as value beholder) are represented as elements of the category of values, and the quality values associated to those options are represented by means of elements of the category of value magnitude.

Applying the definitions of the previous section, we can categories the entities involved in a preferential context as follows. The value of an option $a$ for an agent $i$ is a relational quality that inheres in the value beholder (the role of $i$ ) and that depends on the option $a$ (as value entity). Analogously, the value of an option $b$ for $i$. What counts as a truth-maker of the preferential statement is whether for agent $i$ the value magnitude associated to the value entity of $a$ is greater than the value magnitude associated to the value entity of $b$ (according to the scale provided by the category of value magnitudes).

On this ground, we can assess the placement of preference relations $\operatorname{PREF}(i, a, b)$ among the taxonomy of the previous section.

Firstly, we show that preference relations are not internal, thus they are external relations. Remind that in an internal relations, the individual qualities of the relata are sufficient to assess the value of the statement. In the case of preference relation, the qualities that are required to assess whether $a$ is more valuable than $b$ for $i$ are external qualities that inhere in $i$.

\footnotetext{
4 This simplification amounts to assuming that in a preference statement only one dimension of choice is involved [15,19]. That is, the value of $a$ for $i$ does not depend on any further conditions. To extend this modelling, one may assume that the value of $a$ for $i$ may depend on a number of parameters; e.g. "the value of $a$ for $i$ given that $i$ already has a certain amount of $a$ " may capture the marginal value for $i$ of getting a further $a$.

${ }^{5}$ A specification of this category, and what types of metric spaces is associated to it, is left for future work and for dedicated application of specific views of preferences. We shall also discuss this point in the next section, when we approach the distinction between cardinal and ordinal preferences.

${ }^{6}$ This is the way qualities and quality values are related for instance in DOLCE, cf. $[2]$.
} 
Secondly, preferences are descriptive relations. According to our previous definition, a relation is descriptive if the truth-value of the statement depends on at least one relational quality that depends on one of the other relata. In the case of preference relation, the value of $a$ (or $b$ ) for agent $i$ is indeed a relational quality of $i$ that depends on one of the (other) relata of the preference relation, namely $a$ (or $b$ ).

Finally, preference relations are contingent relations: the mere existence of the relata is not sufficient for assessing the truth-value of $\operatorname{PREF}(i, a, b)$. For instance, the mere existence of $i, a$ and $b$ does not make $a$ or $b$ valuable for her or him, as $i$ may not be aware of their existence, or $i$ never had to compare $a$ and $b$.

Therefore, to conclude, preference relations are external, descriptive, and contingent relations.

\subsection{The truth-makers of statements about preference relations}

In order to assess the truth-value of a statement about preferences, a number of entities must be presupposed. Firstly, we have to postulate the existence of an agent and two alternatives. Secondly, we have to presuppose the existence of a number of relational qualities that inhere in $i$ and that existentially depend on $a$ and $b$. We can define the truth-maker of the preferential statement $\operatorname{PREF}(i, a, b)$ as the (mereological) sum of the relational qualities that inhere in $i$ and that existentially depend on $a$ (the value of $a$ for $i$ ) and of the relational quality that inhere $i$ and depends on $b$ (the value of $b$ for $i$ ). That is, the truth-maker of $\operatorname{PREF}(i, a, b)$ is a complex relational quality that inheres in the agent $i$.

More specifically, we are here endorsing a weak version of truth-making, cf [16]. In the strong version of truth-making $t$ is a truth-maker of the sentence $\phi$ if the existence of $t$ is sufficient to make $\phi$ true. By contrast, $t$ is a weak truthmaker of $\phi$ if it makes the proposition $\phi$ true not just because of its existence, but because of the way $t$ contingently is. The distinction between strong and weak truth-makers is crucial for investigating preference relation.

For $\operatorname{PREF}(i, a, b)$, the mere existence of the sum of the the relational qualities is not sufficient to determine the truth-value of $\operatorname{PREF}(i, a, b)$. One has to look at the quality values, in the category of value magnitude, of the relational qualities "the value of $a$ for $i$ " and "the value of $b$ for $i$ ", and to check their relationship. There are in general four cases: $i$ ) the value magnitude of $a$ is greater than the value magnitude of $b$, and in this case $\operatorname{PREF}(i, a, b)$ is true; $i i)$ the value magnitude of $b$ is greater than the value magnitude of $b$, and in that case $\operatorname{PREF}(i, b, a)$ is true; iii) they are equal (i.e. they are located in the same point of the category of value magnitude), and in that case $\operatorname{both} \operatorname{PREF}(i, a, b)$ and $\operatorname{PREF}(i, b, a)$ hold, according to a weak version of preference (thus $i$ is indifferent between $a$ and $b$ ); $i v)$ they are incomparable, and in that case neither $\operatorname{PREF}(i, a, b)$ or $\operatorname{PREF}(i, a, b)$ are true.

Thus, from an ontological perspective, the preference relation is an interesting type of external contingent non-descriptive relation whose truth-value depends on relational qualities as well as on a comparative relation between value magni- 
tudes. We shall see this aspect in more detail by approaching, in the next section, the formalisation of this view in OntoUML.

\section{An Ontology Module for Preference in OntoUML}

We represent the previous view by starting the development of a module of OntoUML dedicated to preference relations. OntoUML $[9,8]$ is an ontologically well-founded version of the UML 2.0 fragment of class diagrams, which has been extensively used for developing an ontologically sound methodology for conceptual modelling. Moreover, OntoUML has a denotational formal semantics and several operational semantics done by mappings to specific formal languages such as Alloy and Description Logics. To model preferences in OntoUML, we introduce the modelling elements that correspond to the previous analysis. The model is then depicted in Figure 4.

We assume a type for agents AGENT, which classify the entities to which preference relations may be ascribed. AGENT is a mixin (non-sortal), i.e., a type that can classify things of different kinds, i.e., things that have different ontological natures [9]. For instance, in separating between individual and collective preferences, agents may include individual or collective agents, with their specific ontological differences [21].

Since we assume that agents (in the sense of entities with intentionality) are necessarily agents (in the modal sense), we stereotype the type AGENT as a Category, in OntoUML. In other words, a Category is a type that describes essential properties that are shared by entities of different kinds.

Agents can play the "role" of VAlue BEHOLDER in a value ascription relation. Value Ascription is itself an extrinsic and descriptive relation, whose truth-maker is a Value. We represent the type VAlue BeHOLDER as a role mixin because: (i) it classifies entities only contingently, i.e., no value beholder is necessarily a value beholder; (ii) one is a value beholder due to a relational condition; (iii) it is a mixin, i.e., it can classify entities of multiple kinds.

A VALUE (the truth-maker of the value ascription relation ${ }^{7}$ ) is an example of a mode [9]. A mode is an existentially dependent entity that, as such, can only exist by inhering in some other individual. In particular, a value is a relationally dependent mode, i.e., a mode that inheres in an individual but which is also externally dependent on a different individual. In this case, value is a sort of mental state inhering in the value beholder but which is also externally dependent on a VAlue Entity (also termed a Value Bearer). A value mode take a value in at least one (but possibly several) Value Magnitude Spaces. These spaces have, in OntoUML, the semantics of the abstract conceptual spaces, delimiting the possible values an intrinsic property can be projected into [9].

A Preference is also a relational mode but a complex one (i.e., a complex mental state). In fact, a preference is a mode inhering a value beholder that

\footnotetext{
7 The relation of derivation connects a descriptive relation with its truthmaker. In OntoUML, derivation is represented by a dashed line with a black circle in the end connected to the truthmaker type [9].
} 
is essentially composed of exact two existing values, inhering in that very same value beholder. In other words, preference is a mereological sum of values and, in that sense, it is similar to the relators that are the truth-makers of comparative relations [7]. However, since in this case all the constituents of this sum inhere in the same bearer, preference is also an intrinsic dependent entity [9]. In any case, as a relational mode, preference is externally dependent on the value entities on which its constituent value modes depend on (thus, it is a derived relation).

PREFERENCE serves then as the truth-maker of the ternary relation has preference. This relation is itself a derived relation that supervenes on an internal and non-descriptive comparative relation between the VALUE MAGNiTUdES of the value modes that constitute that preference. This, of course, constrains VALUE Magnitude Spaces to be spaces structured by internal comparative relations (in the same manner that spaces such as Weight and Height afford that). Moreover, has preference is an external descriptive relation itself. It connects the rolemixin VALUe BeHOLdER with two other rolemixins PREFERRED ENTITY and Deprecated Entity ${ }^{8}$. The classification condition of Value Entities into playing these "roles" is given by the result of the internal comparative relations between the modes constituting the preference and which are externally dependent on each of these value entities. To put it simply, the value bearer of the value mode that has the highest magnitude in this comparison then plays the role of PREFERREd EnTITY; the value bearer of the one with the lower magnitude then plays the role of DePRECATED EnTITY. It should be clear by now to the reader why both PREFERRED Entity and DePrecated EnTity are modelled as rolemixins: they both classify value entities contingently, in the scope of a (has preference) relation, and classify entities of multiple kinds.

\subsection{Types of preference relations}

The module of OntoUML that we have introduced can be extended to model a variety of types of preference relations that are used in application to specific domains. We mentioned already the distinction between individual and collective preference, that can be approached by refining the class of agents. An articulated analysis of the distinction between individual and collective preferences requires modelling the relationship between the individual and the collective attitude, by analysing how the individuals contributes to the formation of the collective preference, along the lines of the approach in $[20,18]$. We leave this aspect for a future dedicated work.

The distinction between ordinal and cardinal preferences can be approached by refining the value magnitude space. In the ordinal case, we need an ordering structure on the set of quality values representing the value magnitudes. The properties of the preference ordering that are required for capturing the intended view of preference can be derived from the ordering structure of the value

\footnotetext{
${ }^{8}$ Unfortunately, in English, there seems to be no exact term to refer to the nonpreferred entity of a preference relation. In Portuguese, for instance, there exist in the lexicon both the term Preferido (to refer to the preferred entity) as well as the term Preterido (to refer to the non-preferred one).
} 


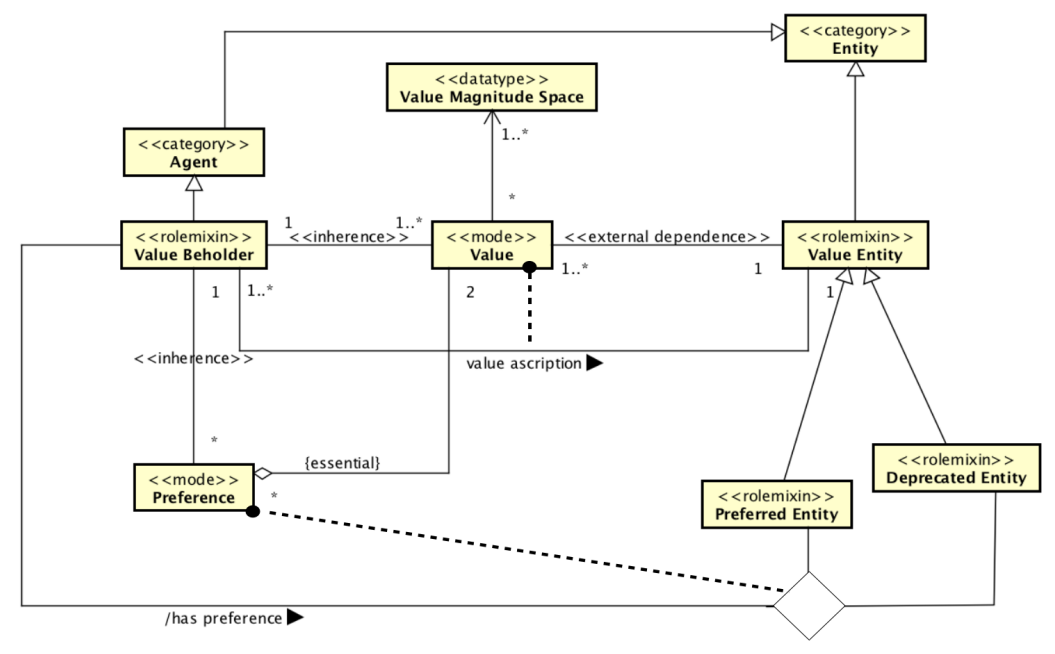

Fig. 1. OntoUML model for preference relations

magnitude space. For instance, if we admit transitive preferences (i.e. relations that satisfy the condition: if $\operatorname{PreF}(i, a, b)$ and $\operatorname{PREF}(i, b, c)$, then $\operatorname{PreF}(i, a, c))$, we need a transitive ordering on the value magnitude space.

The case of cardinal preferences requires the value magnitude space to be constructed as a set of numbers, that provide a measure of the value magnitudes (e.g. the real numbers). The choice of the space depends on the scale and on the granularity assumed to assess the comparisons of value magnitudes.

A further important aspect of preference relation in the economic literature is whether they are comparable or not, cf. [24]. Briefly, two agents' preference relations are comparable if there is common scale that allows for making sense of comparisons such as "the preference of agent $i$ for $a$ over $b$ is more intense than the preference of agent $j$ for $c$ over $d$ ". Comparability is a fundamental concept in economics that allows for enabling a number of important solution concepts in voting, resource allocation, and mechanism design. In our module, comparability can be approached by assuming a local value magnitude space for each individual preference relation. The assumption of comparability would then entail that the value magnitude spaces of distinct individuals are comparable by means of a new relation.

Finally, an important aspect of (cardinal) preference relations and in particular of expected utility theory is the role of probability to model the likelihood of the event in which an agent obtains the objects involved in the preference relation. To include probabilities in our module, we have to provide a more complex construction of the value magnitude space where the value magnitude associated to a certain value also depends on the risk attitudes of the agent involved. We leave this aspect to a future dedicated ontological foundation of expected utility theory. 


\section{Conclusion}

We have presented the basics of an ontological analysis of preference relations. After discussing the type of preference relations within a taxonomy of types of relations, we concluded that preferences are external, descriptive and contingent relations. We investigated the truth-makers of the sentences regarding preferences and we proposed to view them as complex relational qualities that inhere a value beholder. Prosaically, the preference is a complex mental state of an agent.

The type of preference relations is interesting from an ontological perspective because it requires a complex truth-making mechanism that involves both external and internal relations, the latter type enters in the comparisons of the value magnitudes associated to the value entities that are under comparison. Future work shall investigate this mechanism in general to understand which relations meet this pattern.

Then, we presented a formalisation of our view of preferences in OntoUML, providing the essential elements of a dedicated module. The motivation for using the OntoUML methodology is to provide an accessible and sharable modelling of preference relations that may be applied across domains to foster the interoperability and the mutual understanding among modellers. Future work regards the development of the OntoUML module to articulate specific types of preferences and the integration of the preference module with other module designed to treat important concepts in economics, e.g. economic competition [26] and risk [27]. Moreover, we are interested in providing a formalisation of this view of preference in a logic-based language to integrate it with foundational ontologies such as UFO or DOLCE $[9,2]$ and to study reasoners for the preference module.

\section{References}

1. Arrow, K.: Social Choice and Individual Values. Cowles Foundation for Research in Economics at Yale University, Monograph 12, Yale University Press (1963)

2. Borgo, S., Masolo, C.: Foundational choices in dolce. In: Staab, S., Studer, R. (eds.) Handbook on Ontologies. Springer, second edn. (2009)

3. Bratman, M.: Intention, plans, and practical reason. CSLI Publications (1987)

4. Dietrich, F., List, C.: A reason-based theory of rational choice. Nous 47(1), 104-134 (2013)

5. Dietrich, F., List, C.: Where do preferences come from? International Journal of Game Theory 42(3), 613-637 (2013)

6. Gärdenfors, P.: The geometry of meaning: Semantics based on conceptual spaces. MIT Press (2014)

7. Guarino, N., Guizzardi, G.: Relationships and events: towards a general theory of reification and truthmaking. In: Conference of the Italian Association for Artificial Intelligence. pp. 237-249. Springer (2016)

8. Guizzardi, G., Fonseca, C., Benevides, A.B., Almeida, J., Porello, D., Sales, T.: Endurant types in ontology-driven conceptual modeling: Towards OntoUML 2.0. In: 37th International Conference on Conceptual Modeling (ER) (2018) 
9. Guizzardi, G.: Ontological foundations for structural conceptual models. Ph.D. thesis, CTIT, Centre for Telematics and Information Technology, Enschede (2005), http://doc.utwente.nl/50826/

10. Kreps, D.: Notes On The Theory Of Choice. Underground Classics in Economics, Avalon Publishing (1988), https://books.google.it/books?id=9D0Oljs5GrQC

11. List, C., Pettit, P.: Group Agency. The possibility, design, and status of corporate agents. Oxford University Press (2011)

12. MacBride, F.: Truthmakers. In: Zalta, E.N. (ed.) The Stanford Encyclopedia of Philosophy. Metaphysics Research Lab, Stanford University, fall 2016 edn. (2016)

13. Masolo, C., Vieu, L., Bottazzi, E., Catenacci, C., Ferrario, R., Gangemi, A., Guarino, N.: Social roles and their descriptions. In: Principles of Knowledge Representation and Reasoning: Proceedings of the Ninth International Conference (KR2004), Whistler, Canada, June 2-5, 2004. pp. 267-277 (2004)

14. Moore, G.E.: External and internal relations. In: Proceedings of the Aristotelian Society. vol. 20, pp. 40-62 (1919)

15. Ottonelli, V., Porello, D.: On the elusive notion of meta-agreement. Politics, Philosophy \& Economics 12(1), 68-92 (2013)

16. Parsons, J.: There is no truthmakerargument against nominalism. Australasian Journal of Philosophy 77(3), 325-334 (1999)

17. Porello, D.: Ranking judgments in arrow's setting. Synthese 173(2), 199-210 (2010)

18. Porello, D.: A proof-theoretical view of collective rationality. In: IJCAI 2013, Proceedings of the 23rd International Joint Conference on Artificial Intelligence, Beijing, China, August 3-9, 2013 (2013)

19. Porello, D.: Single-peakedness and semantic dimensions of preferences. Logic Journal of the IGPL 24(4), 570-583 (2016)

20. Porello, D.: Logics for modelling collective attitudes. Fundamenta Informaticae 158(1-3), 239-275 (2018)

21. Porello, D., Bottazzi, E., Ferrario, R.: The ontology of group agency. In: Proceedings of the 8th International Conference on Formal Ontology in Information Systems. FOIS 2014, IOS Press (2014), in press

22. Porello, D., Guizzardi, G.: Towards a first-order modal formalisation of the unified foundational ontology. In: Proceedings of the Joint Ontology Workshops 2017 Episode 3: The Tyrolean Autumn of Ontology, Bozen-Bolzano, Italy, September 21-23, 2017. (2017)

23. Rao, A.S., Georgeff, M.P.: Modeling rational agents within a bdi-architecture. KR 91, 473-484 (1991)

24. Roemer, J.E.: Theories of distributive justice. Harvard University Press (1998)

25. Russell, B.: Philosophical Essays. Routledge (1910)

26. Sales, T., Porello, D., Guizzardi, G., Mylopoulos, J., Guarino, N.: Ontological foundations of competition. In: 10th International Conference on Formal Ontologies and Information Systems (FOIS 2018), Cape Town, South Africa, 2018 (2018)

27. Sales, T.P., Baião, F., Guizzardi, G., Guarino, N., Mylopoulos, J.: The common ontology of value and risk. In: 37th Int. Conf. on Conceptual Modeling (ER) (2018)

28. Sales, T.P., Guarino, N., Guizzardi, G., Mylopoulos, J.: An ontological analysis of value propositions. In: 21st IEEE International Enterprise Distributed Object Computing Conference, EDOC 2017, Quebec City, QC, Canada, October 10-13, 2017. pp. 184-193 (2017)

29. Von Neumann, J., Morgenstern, O.: Theory of Games and Economic Behavior. Princeton University Press (1947) 